\title{
MODELING PLASTICITY BY NON-CONTINUOUS DEFORMATION
}

\section{YARON BEN-SHMUEL' ${ }^{1}$ AND ELI ALTUS ${ }^{2}$}

\author{
Faculty of Mechanical Engineering \\ Technion - Israel Institute of Technology \\ Haifa 32000, Israel \\ 1yaronbs@tx.technion.ac.il \\ ²altus@tx.technion.ac.il
}

Key words: Plasticity, Non-continuous deformation, Particle interactions.

\begin{abstract}
Plasticity and failure theories are still subjects of intense research. Yet, no comprehensive theory has been achieved and the Molecular Dynamic method is far from providing a general model which contains only the essentials. This study is motivated by the observation that the continuum assumption in plasticity that neighbour material elements remain neighbours at all-time are physically impossible, since neighbour detachments, local slips and neighbour switching must operate, i.e., Non-Continuous Deformation (NCD). Material microstructure is modelled by a set of point elements (particles) interacting with their neighbours. Each particle can detach from its neighbours and/or attach to a new neighbour during deformation. Simulations on two dimensional specimens subjected to uniaxial compression loading and unloading were conducted. Each specimen contained 100 particles with stochastic heterogeneity controlled by a "disorder" parameter $\lambda$. It was found that a. the macro response is typical to elasto-plastic behaviour; b. The number of detachments is linear with plastic energy; c. The number of attachments is linear with the residual strain, and d. Volume is preserved under plastic deformation. Rigid body displacement of local ensemble of elements was also observed. Higher disorder coefficient $\lambda$ decreases the macro elastic modulus and increases the plastic energy.
\end{abstract}

\section{INTRODUCTION AND MOTIVATION}

Plastic deformation is strongly related to the microstructure. Well known phenomenon such as strain hardening and Bauschinger effect are mostly treated on the continuum basis. The lack of direct correlation between macro parameters and their micro processes makes it seem impossible that continuum theories can offer a physical interpretation for those complexities. Nevertheless, additional terms can reflect micro processes by second-order statistical quantities (point correlations) such as Reynolds stress tensor in Reynolds-averaged Navier-Stokes (RANS) equations ${ }^{[1]}$. Unfortunately this approach has not been materialized for solids.

Granular flow exhibits similar NCD mechanisms. Bazant ${ }^{[2]}$ developed "Spot" model which assumes a specific local collective motion of particles which could be compared to experiments. Classical continuum mechanics was used by Kamrin et al. ${ }^{[3,4]}$ which showed limited capability. Both studies did not relate the model to plasticity in solids. 
There are many studies on double well potential models which exhibit a strain jump in both directions of loading ${ }^{[5,6]}$. However these models not sufficient to describe 2D deformation were material elements can be reorganised during deformation.

Prakash and W. Cleary ${ }^{[7]}$ used the Smooth Particle Hydrodynamics (SPH) method to simulate metal extrusion. Large plastic deformation is observed but macro continuum plastic model is incorporated ("radial return plasticity model").

Molecular Dynamics (MD) simulations can act as a bridge between the micro length and time scales and the macroscopic behaviour ${ }^{[8]}$. Mordehai et al ${ }^{[9]}$ studied the size effect of a single-crystal under compression using MD simulations compared with experimental results (Fig.1).

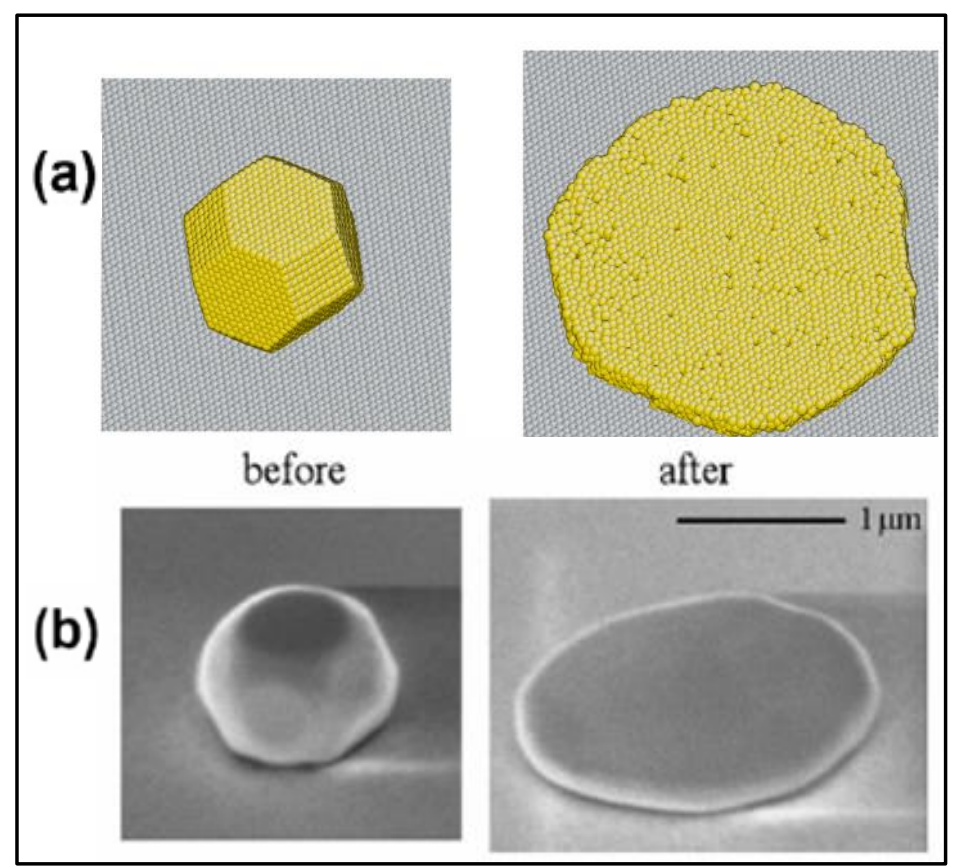

Figure 1: Atomistic ensemble under compression: (a) MD simulation of initially $4.9 \mathrm{~nm}$ size crystal (b) a small $\sim 300 \mathrm{~nm}$ top diameter real crystal before and after deformation (Mordehai et al., 2011).

Since the total amount of atoms per unit area is conserved non-continuous deformation must be admitted. Nano "holes" are also observed. Thus, the NCD is materialized in two forms: attachments and detachments between neighbour atoms. These mechanisms are not included in the classical continuum plasticity theories.

Diffusion based models contains the physical mechanisms of attachments and detachments but their effect is commonly modelled directly on the macro (averaged) level.

\section{MATERIAL MORPHOLOGY}

Material is composed of a set of $N$ "particles" located at $\mathbf{x}^{I}$, where $\left.{ }^{I}\right)$ is the particle's index.

\subsection{Initial configuration}

In the undeformed state the internal and the external forces are zero and the "material" is 
stress free. For the research to be based on fundamental cell distribution, the particle morphology is built on a net containing $n \times m$ cells with dimensions:

$$
a_{1}=n \alpha_{1} ; a_{2}=m \alpha_{2}
$$

where $a_{1}, a_{2}$ are the height and width of a 2D morphology and $n, m$ are the number of cells in the $\boldsymbol{e}_{1}$ and $\boldsymbol{e}_{2}$ direction, respectively.

A single particle is inserted in each cell (constraint a). Its location is controlled by a disorder parameter $0 \leq \lambda \leq 1$ (constraint $b$ ). When $\lambda=0$ all particles are located at the centre of each cell, i.e., fully ordered and deterministic case. When $\lambda=1$ each particle is randomly located anywhere within the boundaries of the cell. This type of microstructure is related to a Voronoi tessellation with the above two constraints.

Fig. 2 demonstrates the initial state morphology as a function of $\lambda$. It includes the interaction between particles which will be discussed in later. Microstructure versatility is clearly observed.

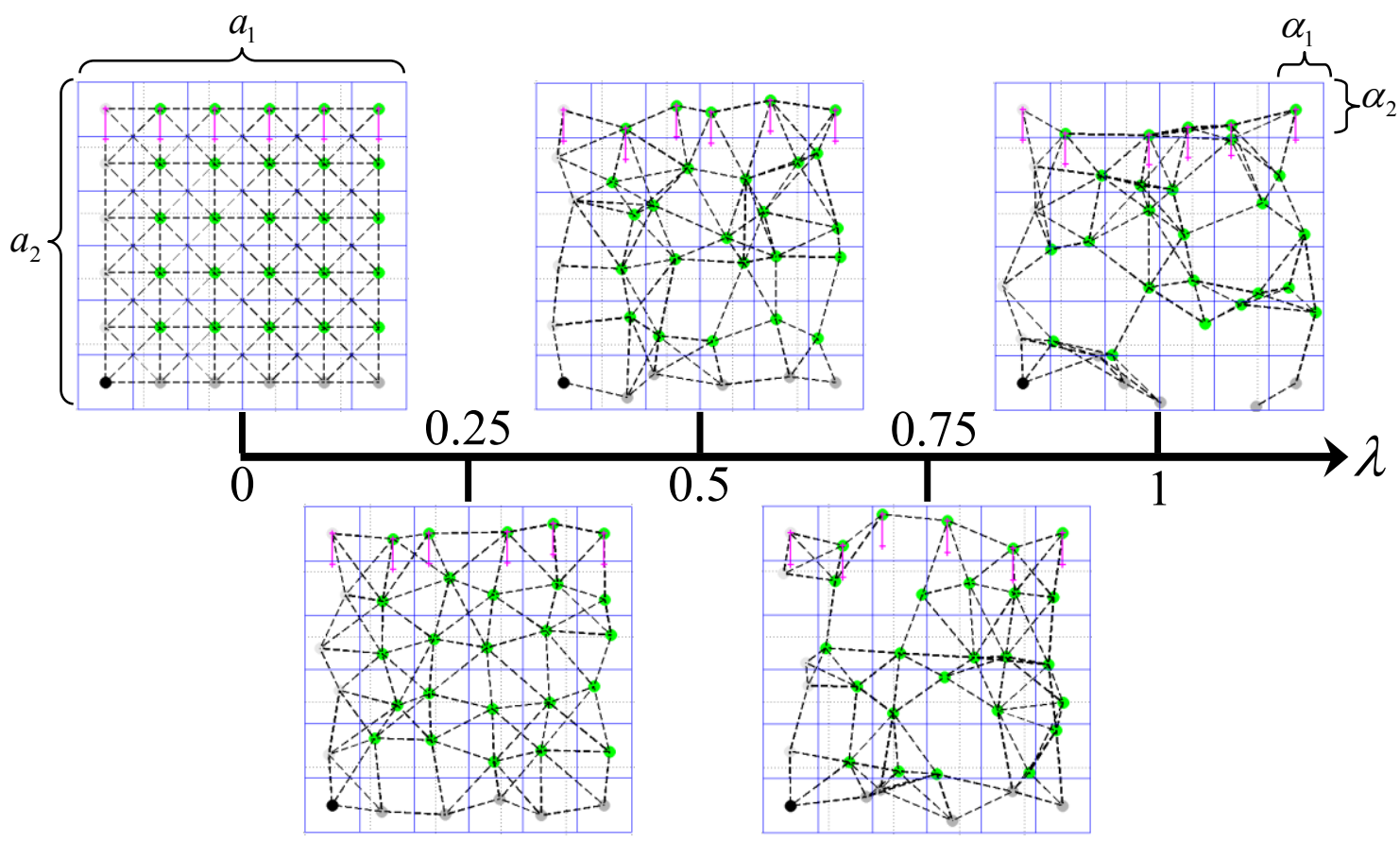

Figure 2: Typical initial configurations of the 2D microstructure for various values of $\lambda$.

\subsection{Particle interactions}

Each particle interacts (force) with its neighbours. The neighbouring particles are located within a circle $R$ which controls the morphological connectivity,

$$
R=C\left(\alpha_{i} \alpha_{i}\right)^{1 / 2}
$$

The radius $R$ is proportional to the diagonal of the cell $(C=1) . C>1$ reflects higher connectivity. In this study $C=1.1$ is chosen to minimize severe discontinuities in the model and still 
allow Attach-Detach (AD) mechanism.

Define $d^{I J}$ as the Euclidean distance between particles $I, J$,

$$
d^{I J}=\left\|x^{I}-x^{J}\right\|_{2}
$$

if $d^{I J} \leq R$ particles $I, J$ are in interaction.

\subsection{Incremental configuration evolution}

There are two possibilities of changing the interaction status of every 2 particles during loading: from non-interacting to interacting ('attachment rule') and vice versa ('detachment rule'). The two rules are sufficient ingredients for our NCD model.

The characteristics of interaction between two neighbouring particles are very similar to the atomistic model, i.e., repulsive and attractive forces. The repulsive force is consistent with the "exclusion principle" and the attractive force act as a 'glue' which keeps the material points together. The specific constitutive relation is not important for our study, and therefore we consider a linear stress-strain law.

Define $d_{0}^{I J}$ as the zero force distance during deformation and the interaction force between particles $I, J$ at some time $t$ as,

$$
f^{I J}=H\left(d_{0}^{I J}(S+1)-d^{I J}\right) k^{I J}\left(d^{I J}, d_{0}^{I J}\right)
$$

$H$ is the Heaviside Function, $S$ is a predefined parameter (strain) which remains constant through the simulation and $k^{I J}$ is a function describing the interaction relation between the two particles.

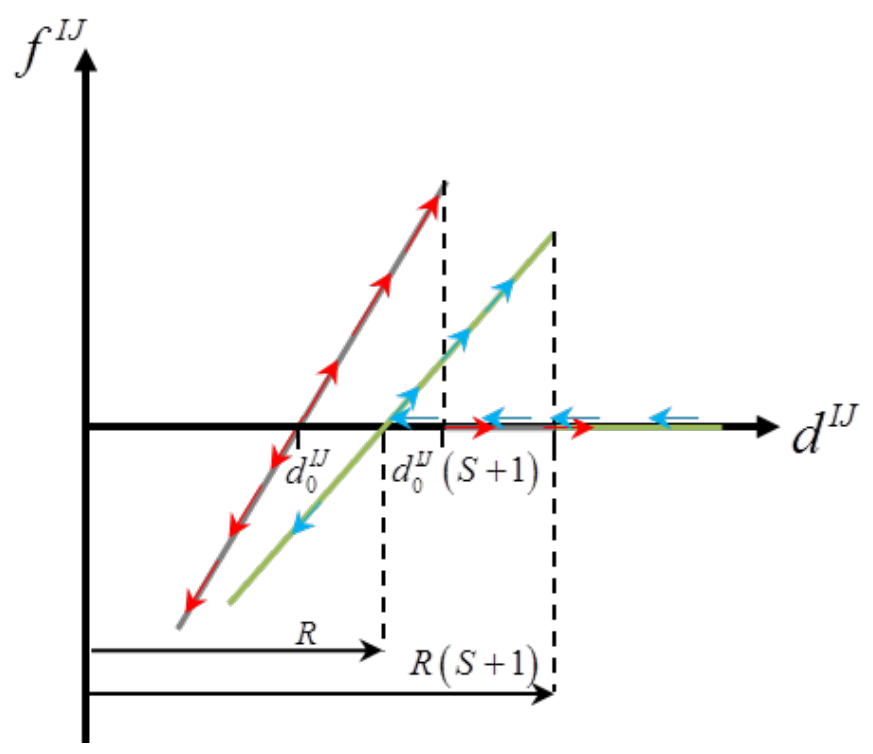

Figure 3: The micro constitutive relation schematically describing the AD mechanism of interaction between two particles.

Fig. 3 shows schematically the basic features of $f^{I J} . d^{I J}=d_{0}{ }^{I J}$ at $t=0$. As $d^{I J}$ increases the force increases (red arrows) until $d^{I J}=d_{0}^{I J}(S+1)$ where particles are no longer in interaction (zero 
force) i.e., detachment. Notice that a correction to $f^{J J}$ is needed to fulfil the 'exclusion principle' e.g., the force must go to $-\infty$ at some threshold. However, in our case the strains are small enough not to reach such an extreme state.

The 'attachment rule' is as follows: two far away particles with no interaction may come close $\left(d^{I J} \leq R\right)$, then the new interaction is based on a zero interaction distance $d_{0}{ }^{I J} \leq R$ (blue arrows).

Note that two interacting particles experiencing detachment (red arrows) are not necessarily the same two particles experiencing the attachment (blue arrows).

\section{CONSTITUTIVE EQUATIONS}

This chapter describes the collective behaviour of the $N$ particles set in a matrix formulation and is the basis for future constitutive macro equations.

\subsection{Mathematical background}

The Hadamard product of two $m \times m$ matrices $\mathbf{A}$ and $\mathbf{B}$ is defined by

$$
(\mathrm{A} \circ \mathrm{B})_{i j}=\mathrm{A}_{i j} \mathrm{~B}_{i j} \quad \forall 1 \leq i, j \leq m,
$$

which is element-wise multiplication [Horn, R., 1994].

The identity matrix $\mathbf{J}$ under Hadamard product is $m \times m$ matrix with all entries equal to 1 .

$$
\mathrm{J}_{i j}=1 \quad \forall 1 \leq i, j \leq m
$$

$\mathbf{A}$ has a Hadamard inverse, denoted by $\hat{\mathbf{A}}$, if and only if $\mathbf{A}_{i j} \neq 0$ for all $1 \leq i, j \leq m$ :

$$
\hat{\mathrm{A}}_{i j}=\left(\mathrm{A}_{i j}\right)^{-1} \text {. }
$$

The Hollow matrix $\mathbf{J}$ ' is defined as,

$$
\mathbf{J}^{\prime} \triangleq \mathbf{J}-\mathbf{I}
$$

where $\mathbf{I}$ is the common identity matrix.

The Euclidean Distance Matrix (EDM) $\mathbf{d}(t)$ contains the complete set of distances between every two particles. $d_{i j}$ denotes the corresponding distance between particles $I, J$ at time $t$,

$$
d_{i j}=d_{j i}=\left\|\mathbf{x}^{I}-\mathbf{x}^{J}\right\|_{2} \geq 0 ; \begin{cases}0, & i=j \\ >0, & i \neq j\end{cases}
$$

Notice that $d_{i j}$ is an element (row $i$, column $j$ ) from a $N \times N$ matrix and $d^{I J}$ is a standalone scalar.

The Primary Euclidean Distance Matrix $(\operatorname{PEDM}) \mathbf{d}_{0}(t)$ is the zero force distance between every pair. At $t=0$,

$$
t=0: \quad \mathbf{d}_{\mathbf{0}} \equiv \mathbf{d}
$$

Define $\mathbf{R}$ and $\mathbf{S}$ related to the 'attachment rule' and 'detachment rule', respectively:

$$
\mathbf{R} \triangleq R \mathbf{J}^{\prime}=\mathbf{R}^{T}
$$




$$
\mathbf{S}=S \mathbf{J}^{\prime}=S^{T}
$$

The interaction indicator matrix $\mathbf{m}(\mathrm{t})$ for any pair is 1 if the corresponding particles are in interaction and 0 if the particles have no interaction. At $t=0 \mathbf{m}$ depends solely on R:

$$
\mathbf{m}(t=0)=H\left(\mathbf{R}-\mathbf{d}_{\mathbf{0}}(t=0)\right)=\mathbf{m}^{T}
$$

$H$ sorts out all pairs with distance $\operatorname{abs}(\boldsymbol{d})>R$. The value of $\mathbf{m}(t)$ will be discussed in the following.

\subsection{Load increments}

In each load (or time) increment a deformation change takes place, where the particles move to new locations $\mathbf{x}(t+d t)$. This is carried out in two stages: elastic continuous deformation and inelastic NCD in the form of "Attach-Detach" (AD) mechanism. In stage one structural morphology remain fixed and $\mathbf{x}(t+d t)$ is obtained from the elastic equilibrium equations and effects $\boldsymbol{d}(t+d t)$. Stage two contains pure attachment or detachment of interactions according to the new locations. $\mathbf{m}(t)$ and $\mathbf{d}_{0}(t)$ are updated with no additional deformation.

The 'detachment rule' formulation in the second stage is:

$$
\mathbf{m}^{-}=H((\mathbf{S}-\mathbf{\varepsilon}(t+d t)) \circ \mathbf{m}(t))
$$

where $\mathbf{m}^{-}$is a matrix containing all the remaining interactions not exceeding $S$ and $\boldsymbol{\varepsilon}$ is the strain matrix given by,

$$
\boldsymbol{\varepsilon}(t+d t)=\left(\mathbf{d}(t+d t)-\mathbf{d}_{\mathbf{0}}(t)\right) \circ \hat{\mathbf{d}}_{\mathbf{0}}^{\prime} ; \mathbf{d}_{\mathbf{0}}^{\prime}=\left(\mathbf{d}_{\mathbf{0}}(t)+\mathbf{I}\right)
$$

In (14) the Hadamard product with $\mathbf{m}(t)$ act as a filter to cancel out all those non feasible interactions.

The 'attachment rule' formulation in the second stage is: all the non-interacting particles which admit $\left(d_{i j}<R\right)$ are in interaction with updated $\mathbf{d}_{0}$. This is done by first subtracting $\mathbf{m}(t)$ from all possible interactions and then employ the rule on the remaining interactions,

$$
\mathbf{m}^{+}=H\left((\mathbf{R}-\mathbf{d}(t+d t)) \circ\left(\mathbf{J}^{\prime}-\mathbf{m}(t)\right)\right)
$$

The updated interaction matrix is now given by the sum of (14) and (16),

$$
\mathbf{m}(t+d t)=\mathbf{m}^{-}+\mathbf{m}^{+}=H((\mathbf{S}-\mathbf{\varepsilon}(t+d t)) \circ \mathbf{m}(t))+H\left((\mathbf{R}-\mathbf{d}(t+d t)) \circ\left(\mathbf{J}^{\prime}-\mathbf{m}(t)\right)\right)
$$

Thus, the two stages are assumed independent and can be linearly superposed, which is the simplest approximation.

In the case where $\mathbf{m}^{+} \neq 0$, new interactions are formed with zero strain which means there is no additional elastic (free) energy. This ensures positive dissipative energy of the plastic deformation. The $\mathbf{d}_{0}$ matrix is updated as,

$$
\mathbf{d}_{\mathbf{0}}(t+d t)=\mathbf{d}_{\mathbf{0}}(t)+\mathbf{m}^{+} \circ\left(\mathbf{d}(t+d t)-\mathbf{d}_{\mathbf{0}}(t)\right)
$$

Notice that when $\mathbf{m}^{+}=0, \mathbf{d}_{0}$ remains unchanged as expected. 


\subsection{Tangent stiffness matrix (local and global)}

In this section we explore the relationship between $\mathrm{AD}$ mechanism and the stiffness matrix in order to build a basis for future macro non-continuous model.

Each interaction has a distinct local stiffness matrix $\boldsymbol{k}^{I J}[2 n \times 2 n]$, where $n$ is the dimension of the problem (here $n=2$ ):

$$
\mathbf{k}^{I J}=\mathbf{k}_{E}^{I J}+\mathbf{k}_{G}^{I J}=\frac{c_{0}}{d_{0}^{I J}} \mathbf{G}^{I J}+\frac{f^{I J}}{d^{I J}}\left(\mathbf{A}^{T} \mathbf{A}-\mathbf{G}^{I J}\right)
$$

$c_{0}$ is the intensity of the interaction which is constant and the same for all interactions. The matrix $\mathbf{G}$,

$$
\mathbf{G}^{I J} \triangleq\left(\mathbf{n}^{I J} \mathbf{A}\right)^{T}\left(\mathbf{n}^{I J} \mathbf{A}\right)
$$

where,

$$
\mathbf{n}^{I J}=\frac{1}{d^{I J}}\left(\mathbf{x}^{I}-\mathbf{x}^{J}\right) ; \quad I \neq J
$$

is the unit vector indicating the orientation of interaction at time $t$ and $\mathbf{A}$ is simply,

$$
\mathbf{A}=\left[\begin{array}{ll}
-\mathbf{I}_{[n \times n]} & \mathbf{I}_{[n \times n]}
\end{array}\right]
$$

(19) indicates that $\mathbf{k}^{I J}$ splits naturally into two parts which are called the material stiffness matrix and the geometric stiffness matrix, respectively, in the literature ${ }^{[10]}$. Notice that when $f^{I J}=0$ (attachment), the geometric stiffness matrix vanishes. (19) also expresses the local stiffness matrix for $1 \leq I, J \leq N$. However only few interactions exist in the current configuration and a suitable entry from $\mathbf{m}(t)$ is needed:

$$
\mathbf{k}^{I J}=\left(m_{i j} \mathbf{I}\right)\left(\frac{c_{0}}{d_{0}^{I J}} \mathbf{G}^{I J}+\frac{f^{I J}}{d^{I J}}\left(\mathbf{A}^{T} \mathbf{A}-\mathbf{G}^{I J}\right)\right)
$$

This ensures that only existing interactions will contributes to the global tangent stiffness matrix $\mathbf{K}_{\mathbf{t}}$. The AD mechanism is expressed through (23) via $m_{i j}$ and $d_{0}^{I J}$ : attachment "equals" an increase in the global stiffness and vice versa for detachment.

The global stiffness matrix $\mathbf{K}_{\mathbf{t}}[2 N \times 2 N]$ ( 2 degrees of freedom per particle) is the sum of contributions from all interactions:

$$
\mathbf{K}_{\mathbf{t}}=\sum_{I=1}^{N} \sum_{J=1}^{N}\left(\mathbf{C}^{T} \mathbf{k} \mathbf{C}\right)^{I J}
$$

where $\mathbf{C}^{I J}[2 n \times 2 N]$ is an indicator matrix which transforms the elements of $\mathbf{k}^{I J}$ into the global stiffness matrix in a systematic procedure describe in many books ${ }^{[11]}$ :

$$
\mathbf{C}^{I J}=\left\{\begin{array}{cc}
C_{1,2 I-1}=C_{2,2 I}=C_{3,2 J-1}=C_{4,2 J}=1 \\
0, & \text { all others }
\end{array}\right\} .
$$




\section{SIMULATION AND RESULTS}

\subsection{Problem description and boundary conditions}

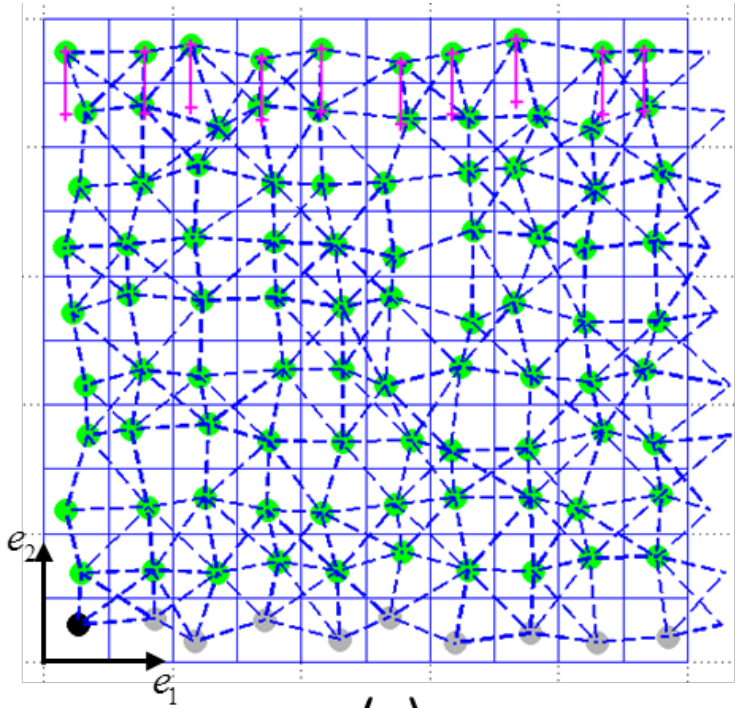

(a)

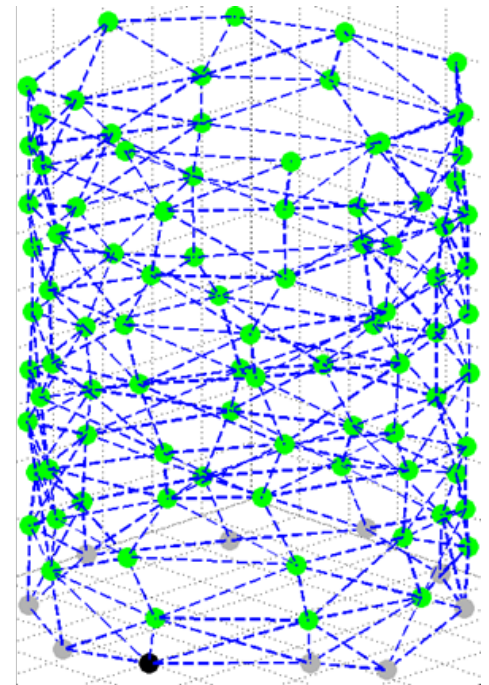

(b)

Figure 4: Typical initial configuration $(\lambda=0.2)$ containing 100 particles: a) 2D microstructure subjected to compression load (pink arrows). Particle at the bottom left corner (black) is fixed to prevent rigid body motion, b) the same 2D microstructure projected on a cylinder, demonstrating PBC.

Consider typical morphology for $\lambda=0.2$ at $(t=0)$ as shown in Fig.4. (10x10) particles are connected by "truss elements" having stiffness according to (4). External force is evenly distributed between all particles in the upper edge throughout the simulation (pink arrows in Fig.4a). Lower edge is controlled by the Boundary Conditions:

$$
u_{2}=0, \sigma_{12}=0
$$

In order to avoid "surface effects", we impose Periodic Boundary Conditions (PBC) as follows: the left and right edges are "stitched" together to form a cylinder-like structure as seen in Fig.4b. The proper "stitching space" is calculated by finding the equilibrium point at which the sum of the horizontal forces is zero.

The above simulates the macro conditions:

$$
\sigma_{11}^{M}=0, \sigma_{22}^{M}=\sigma_{c}
$$

Under the ergodic assumption, prediction of the macro behaviour is done by calculating the ensemble average of at least 200 specimens for each $\lambda$. Thus, smooth macro stress-strain curve is obtained. 


\subsection{Results}

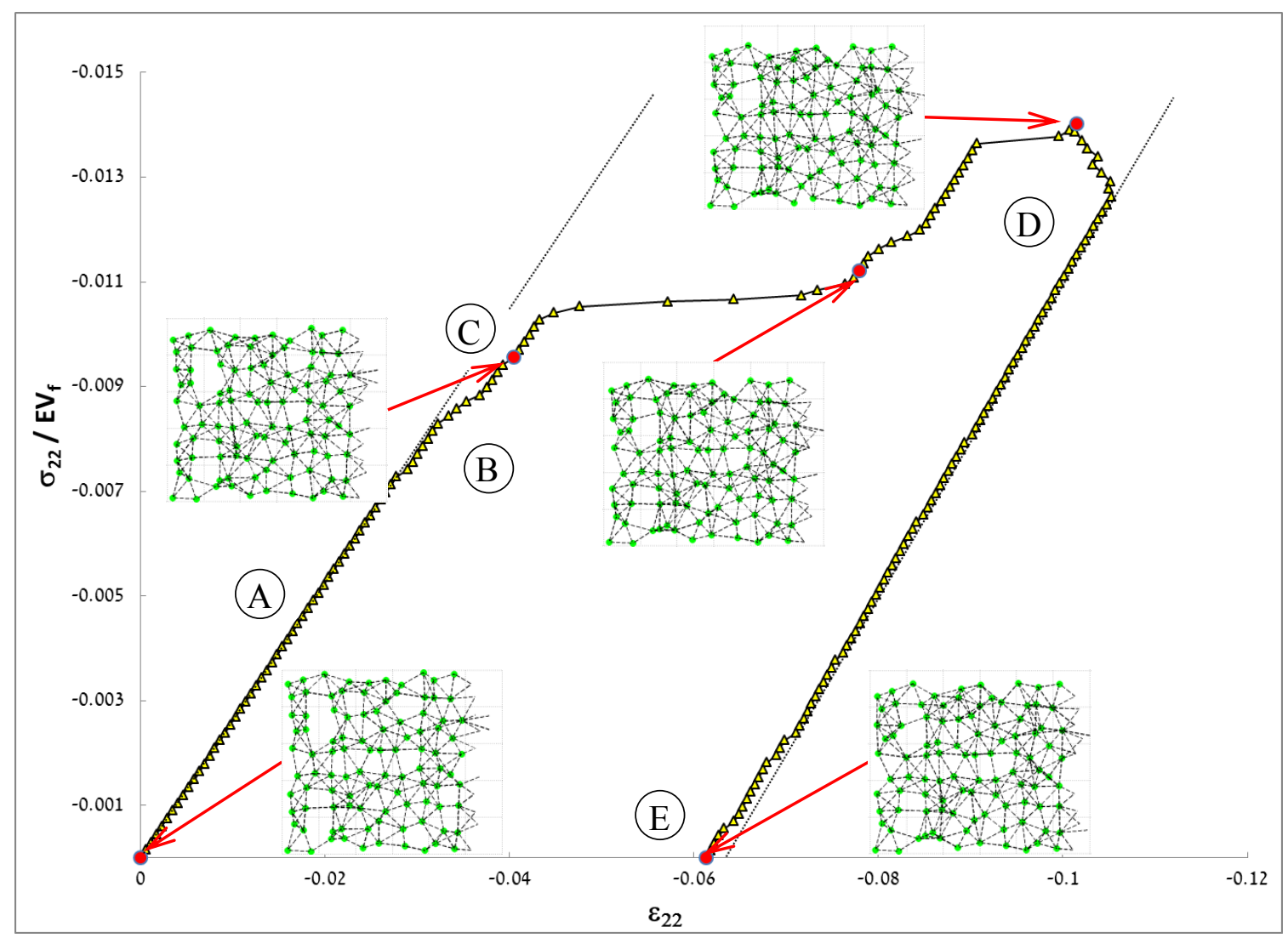

Figure 5: Typical true stress-strain curve $(\lambda=0.5)$ of single specimen under uniaxial compression, $\sigma_{\mathrm{c}}=-0.015$. $\sigma_{22}$ is normalized by $V_{f} E$, where $\mathrm{E}$ is the elastic modulus of a single interaction and $V_{f}$ is the $(\lambda=0)$ volume fraction as seen in Fig. 2.

A typical stress-strain curve of a single specimen is plotted in Fig.5 $(\lambda=0.5)$. Selected configurations at several loading \& unloading stages are shown. At the first part of the loading phase (A) the structure deforms linearly elastic. At a certain loading point the 'detachment' criteria (14) is reached resulting in decreasing stiffness of the structure i.e., yielding (B). The morphology changes and new interactions appear partially restoring the structure's stiffness (C) and the whole process is repeated. At the unloading phase (D-F) the structure deforms elastically with approximately the same modulus. At the end of the unloading phase (E) a slight stiffness decrease is observed due to local detachments. It was found that the Poisson ratio at $(\mathrm{A})$ is $v=0.36$ and at $(\mathrm{E})$ is $(0.066 / 0.061) \cong 1$. Noticing that $(\mathrm{E})$ contains essentially the net plastic deformation, we see that the result complies with the macro volume preservation postulate under plastic deformation.

Fig.6 shows the ensemble average (macro) stress-strain curves for several values of $\lambda$. All specimens were subjected to the same load history i.e., compression loading and unloading. The stress-strain curves demonstrate typical elasto-plastic behaviour. 
It can be seen that:

1. There is a correlation between morphology disorder $(\lambda)$ and the structure's stiffness as well as residual plastic strain $\left(\varepsilon_{\mathrm{p}}\right)$. As $\lambda$ increases the structure's stiffness decreases and the plastic deformation initiates at an earlier stage.

2. From (23) and (24) we conclude that increasing number of interactions also means increasing the stiffness of the structure. In order to gain insight relations between $\lambda$, average number of interactions $<\mathrm{M}>$ and the elastic stiffness $\left\langle\mathrm{E}_{22}>\right.$ we have calculated $<\mathrm{M}>$ and $\left\langle\mathrm{E}_{22}>\right.$, both at the initial configuration, as a function of $\lambda$ (see Fig.7). Notice the approximately linear dependence between $\langle M\rangle$ and $\lambda$.

3. Increasing $\lambda$ also leads to wider range of plasticity, suggesting that plastic energy strongly influenced by material morphology, more specifically to the level of ordering.

4. Unloading is composed of three stages: (A), (B) and (C).

In stage (A) an artificial behaviour is observed. It was found that this phenomenon diminishes when loading increments are sufficiently small. It is concluded that the source of this abnormality is numerical only. The slope in stage (B) is not affected by $\lambda$.

Stage (C) reflects the detachment mechanism emphasized in the loading direction. This behaviour resembles the well-known "Bauschinger effect".

5. The AD mechanism depends on the loading direction and reflects non-isotropic behaviour.

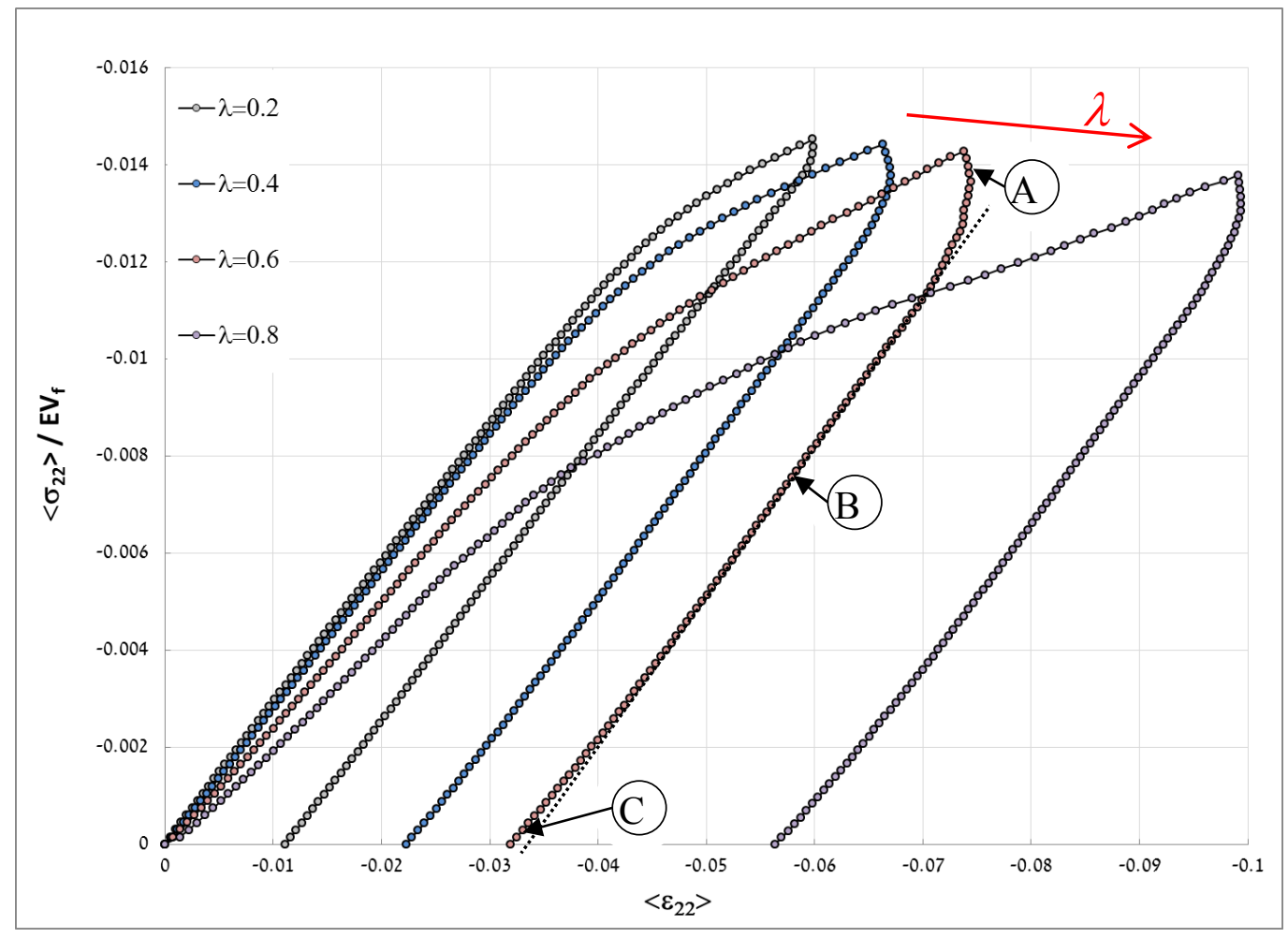

Figure 6: True stress-strain curves for several values of $\lambda$ ranging from 0.2 to 0.8 . Results obtained under uniaxial compression with $\sigma_{\mathrm{c}}=-0.015$. 


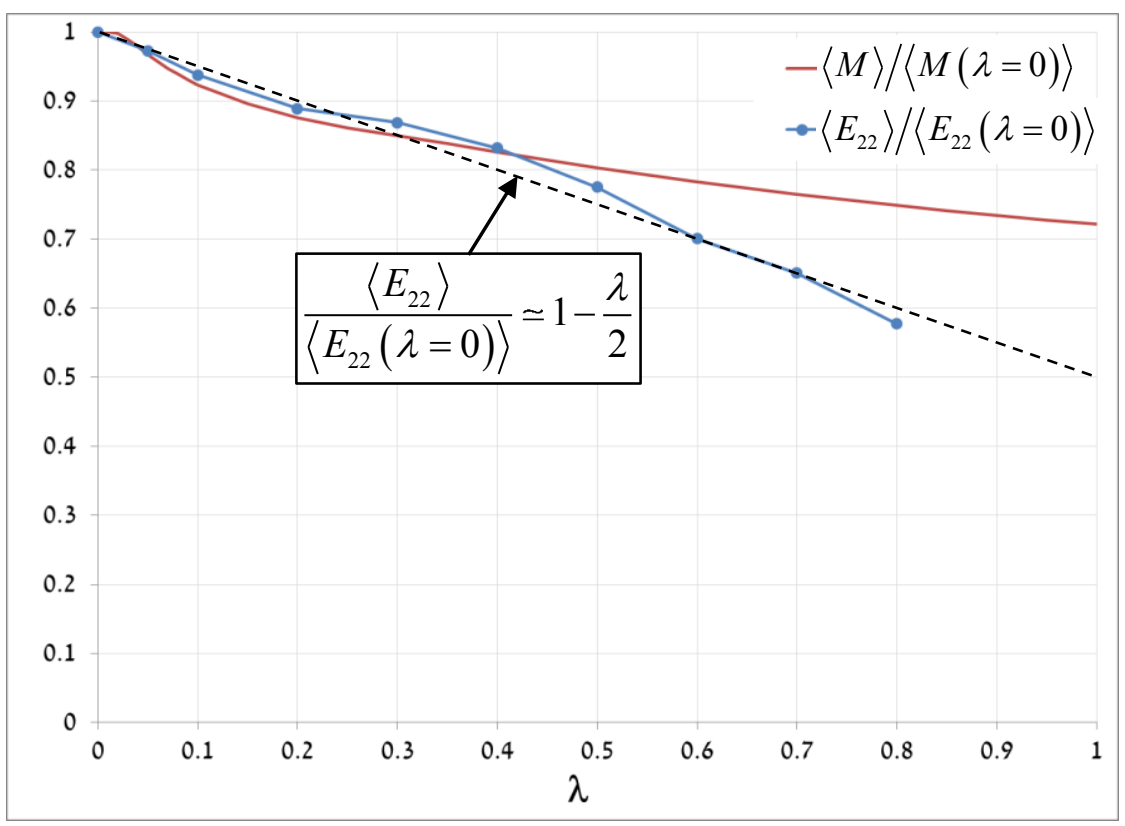

Figure 7: Trends of the elastic modulus $\left\langle E_{22}>\right.$ and number of interactions $\langle M>$ as a function of the disorder parameter $\lambda$.

Fig 8 exhibits the two main correlations between the AD micro mechanisms and the plastic behaviour. Fig.8a shows that plastic energy is essentially linear with the accumulative number of detachments. The effect of $\lambda$ decreases asymptotically for $\lambda \rightarrow 1$. Fig. $8 \mathrm{~b}$ shows typical linear correlations between residual (after unloading) plastic strain and accumulative number of attachments. The aim is to find these coefficients analytically in following studies.

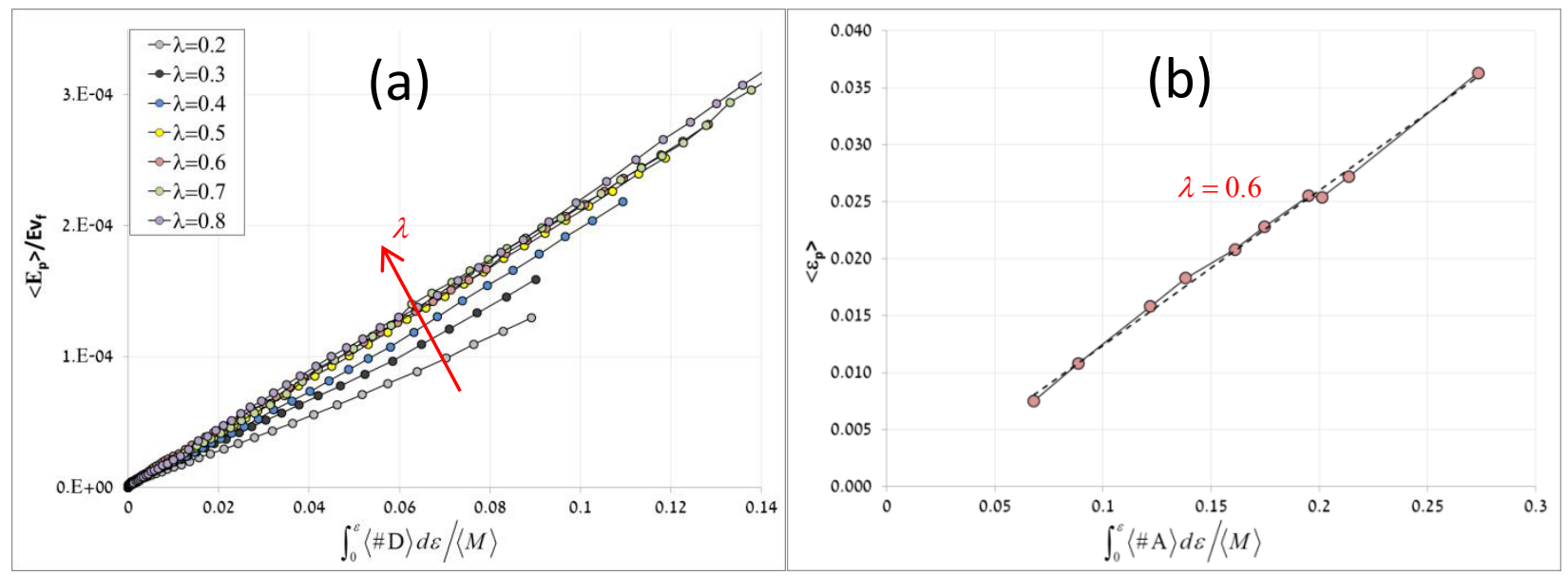

Figure 8: a) Plastic energy vs. accumulative average number of detachments for several values of $\lambda$. b) Residual plastic strain vs. accumulative average number of attachments, for $\lambda=0.6$. 


\section{CONCLUSIONS}

- It is demonstrated that macro plastic deformation can be modelled as an accumulation of local (micro) non-continuous deformation based on particle detachments and attachments.

- A linear correlation is revealed between macro plastic energy and number of detachments, and between residual plastic strain and the number of attachments.

- The micro incremental procedure used in this study, i.e., elastic step followed by inelastic one is consistent with the macro strain decomposition i.e., $\varepsilon=\varepsilon_{e}+\varepsilon_{p}$ used in classical continuum plasticity. Both assume the deformation is the superposed sum of elastic and inelastic parts. This assumption is a $1^{\text {st }}$ order approximation of the general elastic-plastic interaction.

\section{REFERENCES}

[1] McDonough, J. M. [2004], Introductory Lectures on Turbulence. Physics, Mathematics and Modeling, Departments of Mechanical Engineering and Mathematics, University of Kentucky.

[2] Bazant, M. Z. [2006], The spot model for random-packing dynamics. Mechanics of Materials, Vol. 38, pp. 717-731.

[3] Kamrin, K., Rycroft, C. H. and Bazant, M. Z. [2007], The stochastic flow rule: a multiscale model for granular plasticity. Modelling Simul. Mater. Sci. Eng. 15 S449-S464.

[4] Kamrin, K. [2008], Stochastic and Deterministic Models for Dense Granular Flow. Ph.D. thesis, MIT.

[5] Salman, O. U., Truskinovsky, L. [2012], On the critical nature of plastic flow: One and two dimensional models. International Journal of Eng. Sci., Vol. 59, pp. 219-254.

[6] Benichou, I., Givli, S. [2013], Structures undergoing discrete phase transformation. Journal of the mechanics and physics of solids, Vol. 61, pp- 94-113.

[7] Prakash, M., Cleary, P. W. [2015], Modelling highly deformable metal extrusion using SPH. Comp. Part. Mech., doi:10.1007/s40571-015-0032-0.

[8] Allen, M. P. [2004], Introduction to Molecular Dynamics Simulation. NIC Series, Vol. 23, ISBN 3-00-012641-4, pp. 1-28.

[9] Mordehai, D., Lee, S. W., Backes, B., Srolovitz, D. J., Nix, W. D., Rabkin, E. [2011], Size effect in compression of single-crystal gold microparticles. Acta Materialia 59, 5202-5215.

[10] Argyris, J. H., Balmer, H., Doltsinis, J.St., Dunne, P. C., Haase, M., Kleiber, M., Malejannakis, G. A., lejnek, H. -P., Müller, M., Scharpf, D. W. [1979], Finite element method - the natural approach. Computer Methods in Applied Mechanics and Engineering, Vol. 17-18, pp. 1-106.

[11] Bathe, K. -J. [1982], Finite element procedures in engineering analysis. Prentice-Hall, New Jersey. 